\title{
Tip Ton
}

Nach dem Betreten einer Bibliothek verwandelt sich der aufrecht schreitende Mensch - der Biologielehrer sprach vom "homo erectus" - in einen sich der stundenlangen Sitzhaltung mit gekrümmtem Rücken hingebenden "homo bibliothecarius sedutus"1. Mangelnde Bewegung und starres Sitzen schaden bekanntlich nicht nur dem Körper. Als Form zeitgemäßen Lernens empfehlen Mediziner und Ergonomen hingegen ein dynamisches Sitzen, bei dem die Position verändert werden kann, was die Durchblutung fördert und durch erhöhte Sauerstoffzufuhr zu einer besseren Konzentrationsfähigkeit führt.

Für die über 300 Benutzerarbeitsplätze im Erweiterungsbau der Württembergischen Landesbibliothek wurde deshalb ein Stuhl ausgewählt, der auf den ersten Blick - schwarzer Kunststoff, in einer Form durchgezogen - eher schlicht wirkt. Aber "Tip Ton“2 hat es, wie modernes Design oft, in sich. Das bemerkt der darauf Sitzende jedoch erst, nachdem er Platz genommen hat. Der Stuhl bietet ihm zwei Sitzpositionen an: 1. aufrecht, gerade und 2. gekippt, nach vorne geneigt. Die zweite Variante ermöglichen die im vorderen Teil um einen Winkel von 9 Grad abgeschrägten Beinkufen.

Entworfen haben diesen Kippstuhl die britischen Designer Edward Barber und Jay Osgerby. Sie erhielten 2008 den Auftrag, für die Kunstakade- mie der englischen Stadt Tipton nahe Birmingham eine Einrichtung zu kreieren, die der Lernsituation von Schülerinnen und Schülern nach modernen pädagogischen Gesichtspunkten Rechnung tragen sollte. Damit rückte eine neue Ausgestaltung vor allem der Sitzmöbel sofort in den Fokus der Konstrukteure und der Anspruch, ein möglichst gesundes Sitzerlebnis für die Rücken der jungen Menschen zu entwickeln. Wissenschaftliche Unterstützung holten sich Barber \& Osgerby in der Schweiz. Etwa zeitgleich widmete sich eine Studie an der Eidgenössischen Technischen Hochschule (ETH) Zürich in Zusammenarbeit mit dem Möbelhersteller Vitra dem Sitzen in Bewegung auf Bürostühlen.

Die Forschungsergebnisse zeigten die positiven Auswirkungen auf die Bauch- und Rückenmuskulatur bei einer nach vorne geneigten Körperhaltung.

Von der Idee des bewegten Sitzens bis zur Umsetzung in ein fertiges Produkt vergingen Jahre. Dem Gestaltungsprozess folgten zahlreiche, wohl über 30 Entwürfe. 2011 präsentierten die Designer ihren Stuhltyp mit der Möglichkeit, über die vorwärts kippende Positionsänderung in eine zweite Sitzvariante zu wechseln. Und das Kippen lädt wie automatisch zum Wippen ein. Das hält wach und Rückenbeschwerden auf Abstand.

Für die Besucherinnen und Besucher an den Arbeitsplätzen der Neuen WLB eröffnet Tip Ton das Ende statischen Lernens: Nehmen Sie Platz und bleiben Sie - für Körper und Geist - in Bewegung. 